\title{
BRIGHT STARS AND RECENT STAR FORMATION IN THE IRREGULAR MAGELLANIC GALAXY NGC $2366^{1}$
}

\section{A. Aparicio, J. Cepa, C. Gallart, ANd H. O. Castañeda}

Instituto de Astrofísica de Canarias, E-38200, La Laguna, Tenerife, Canary Islands, Spain Electronic mail: aaj@iac.es, jcn@iac.es, cgg@iac.es, hoc@iac.es

\section{CHIOSI}

Dipartimento di Astronomia dell'Università di Padova, Vicolo dell'Osservatorio 5, I-35122, Padova, Italy Electronic mail: chiosi@astrpd.pd.astro.it

\section{G. BERTELLI}

Consiglio Nazionale delle Ricerche, Vicolo dell'Osservatorio 5, I-35122, Padova, Italy Electronic mail: bertelli@astrpd.pd.astro.it

\section{J. M. MAS-HESSE}

Laboratorio de Astrofísica Espacial y Física Fundamental, Apdo. 50727, E-28080, Madrid, Spain Electronic mail: mm@laeff.esa.es

\section{MUÑOZ-TUÑóN}

Instituto de Astrofísica de Canarias, E-38200, La Laguna, Tenerife, Canary Islands, Spain Electronic mail: cmt@iac.es

\section{E. TELLES}

Royal Greenwich Observatory, Madingley Road, Cambridge CB3 OEZ, United Kingdom Electronic mail: etelles@mail.ast.cam.ac.uk

\section{G. TENORIO-TAGLE}

Instituto de Astrofísica de Canarias E-38200, La Laguna, Tenerife, Canary Islands, Spain Electronic mail: gtt@iac.es

\section{A. I. DiAZ AND L. M. GarCíA-VARGaS}

Departamento de Física Teórica, C-XI, Universidad Autónoma de Madrid, Cantoblanco, E-28049, Madrid, Spain Electronic mail: angeles@astrol.ft.uam.es, mansa@astrol.ft.uam.es

\section{F. GARZóN}

Instituto de Astrofísica de Canarias, E-38200, La Laguna, Tenerife, Canary Islands, Spain Electronic mail: fgl@iac.es

\section{R. M. GONZÁLEZ-DELGADO}

Instituto de Astrofísica de Canarias, E-38200, La Laguna, Tenerife, Canary Islands, Spain, and Instituto de Astrofísica de Andalucía, Apdo. 3004, E-18080, Granada, Spain

Electronic mail: rosa@iaa.es

\section{E. PÉREZ}

Instituto de Astrofísica de Andalucía, Apdo. 3004, E-18080, Granada, Spain Electronic mail: eperez@iaa.es

\section{J. M. RODRÍGUEZ-ESPINOSA}

Instituto de Astrofísica de Canarias, E-38200, La Laguna, Tenerife, Canary Islands, Spain Electronic mail: jre@iac.es

\section{E. Terlevich and R. J. Televich}

Royal Greenwich Observatory, Madingley Road, Cambridge CB3 OEZ, United Kingdom Electronic mail: et@mail.ast.cam.ac.uk, rjt@mail.ast.cam.ac.uk 


\title{
A. M. VARela AND J. M. VillcheZ
}

Instituto de Astrofísica de Canarias E-38200, La Laguna, Tenerife, Canary Islands, Spain Electronic mail: avp@iac.es, jvm@iac.es

Received 1995 January 13, revised 1995 March 24

\begin{abstract}
The stellar content of the Im galaxy NGC 2366 is discussed on the basis of CCD $B V R$ photometry. The three brightest blue and red stars have been used to estimate its distance, obtaining a value of $2.9 \mathrm{Mpc}$. The spatial distribution of the young stellar population is discussed in the light of the integrated color indices and the color-magnitude diagrams of different zones of the galaxy. A generalized star formation burst seems to have taken place about $50 \mathrm{Myr}$ ago. The youngest stars are preferentially formed in the South-West part of the bar, where the giant H II complex NGC 2363 is located, being younger and bluer. The bar seems to play a role favoring star formation in one of its extremes. Self-propagation however, does not seem to be triggering star formation at large scale. A small region, populated by very young stars has also been found at the East of the galaxy. (c) 1995 American Astronomical Society.
\end{abstract}

\section{INTRODUCTION}

A considerable effort is being devoted to the analysis of the stellar content of nearby galaxies through the photometry of their resolved stars. These data, combined with spectroscopic and radio observations, provide important information about the history of the star formation and the processes of the star formation in galaxies. The understanding of these processes is relevant, since they define the path followed by the galaxy in its evolution.

In this context, late type galaxies, in particular, irregulars, are relevant for a number of reasons:

(1) They are relatively simple objects, at least from the point of view that they do not show complex spiral-like structures. They are also relatively small systems. These characteristics make easier the photometric analysis of the resolved stars. It is, in principle, possible to measure virtually all the stars down to a certain magnitude, so having direct information about the corresponding interval of stellar masses and ages.

(2) They are objects with a high activity of star formation. This means that a relatively large number of young bright and, therefore, resoluble stars is present in them.

(3) They are relatively young objects, in the sense that they show usually low abundances of heavy elements and a large amount of gas. Because of this, the study of these galaxies provides information about the star formation processes in the first evolutionary steps of stellar systems.

Our project is devoted to the analysis of the stellar content in nearby galaxies. It is included in the GEFE ${ }^{2}$ program. In

\footnotetext{
${ }^{1}$ Based on observations made with the Isaac Newton-Telescope operated on the island of La Palma by the Royal Greenwich Observatory in the Spanish Observatorio del Roque de los Muchachos of the Instituto de Astrofísica de Canarias.

${ }^{2}$ The main aim of the GEFE (Grupo de Estudios de Formación Estelar) project is the characterization of the star formation mechanisms in galaxies. GEFE obtained $5 \%$ of the total observing time of the telescopes of the Observatories of the Instituto de Astrofísica de Canarias. This time is distributed by the Comité Cientifico Internacional among international programs.
}

TABLE 1. Global parameters for NGC 2366.

\begin{tabular}{|c|c|c|}
\hline$\alpha_{1950}$ & $07^{\mathrm{h}} 23^{\mathrm{m}} 37^{\mathrm{s}}$ & \\
\hline$\delta_{1950}$ & $+69^{\circ} 19^{\prime} .1$ & \\
\hline$l_{1950}:$ & $146^{\circ} .43$ & \\
\hline$b_{1950}$ & $+28^{\circ} .54$ & \\
\hline$m_{B}^{b_{2}}$ : & $11.03-11.12$ & 1,2 \\
\hline$m_{V}^{b i}:$ & 10.62 & 3 \\
\hline$m_{B}^{b i}:$ & 10.74 & 1 \\
\hline$m_{I}^{h i}:$ & 10.82 & 1 \\
\hline$m_{H}^{b i}:$ & 10.78 & 1 \\
\hline$A_{B}=A_{B}^{b}+A_{B}^{i}$ & $0.47-0.52$ & $1,3,4,5$ \\
\hline $\begin{array}{lll}B & B & -\end{array}$ & $63^{\circ}-69^{\circ}$ & 1,10 \\
\hline$(m-M)_{0}:$ & $27.07-27.65$ & $6,7,8,9$ \\
\hline$W_{20}$ : & $113-118 \mathrm{Kms}^{-1}$ & $1,10,11$ \\
\hline$W_{50}$ : & $96 \mathrm{Kms}^{-1}$ & 11 \\
\hline$v_{L G}$ : & $285 K m s^{-1}$ & 10 \\
\hline$M_{T}:$ & $(1.8-4.6) \times 10^{9} M_{\odot}$ & 10,12 \\
\hline$M_{H I} / M_{T}$ & $0.33-0.48$ & 10,12 \\
\hline$M_{T} / L_{B}$ & 2.4 & 8 \\
\hline $12+\log (O / H)$ & $7.81-8.02$ & $13,14,15$ \\
\hline
\end{tabular}

Notes: $\alpha_{1950}, \delta_{1950}$ : equatorial coordinates; $l_{1950}, b_{1950}$ : galactic coordinates; $m_{B}^{b i}, m_{V}^{b i}, m_{R}^{b i}, m_{I}^{b i}, m_{H}^{b i}$ : apparent magnitudes corrected for galactic and internal extinction; $A_{B}$ : total (galactic and internal) extinction in $B ; i$ : inclination angle; $(m-M)_{0}$ : true distance modulus; $W_{20}, W_{50}$ : line width of the $21 \mathrm{~cm}$ HI line measured respectively at $20 \%$ and $50 \%$ of peak intensity; $v_{L G}$ : velocity relative to the Local Group barycenter, using the Yahil, Tammann \& Sandage's (1977) correction for the solar motion; $M_{T}$ : total mass; $M_{H I} / M_{T}$ : Hydrogen to total mass ratio; $M_{T} / L_{B}$ : mass-luminosity relation; $12+\log (O / H)$ : Oxygen abundance relative to Hydrogen.

References: (1) Pierce \& Tully 1992; (2) Kraan-Korteweg et al 1988; (3) Shimasaku \& Okamura 1992; (4) de Vaucouleurs et al 1991; (5) Burstein \& Heiles 1984; (6) Tikhonov et al 1991; (7) Sandage \& Tammann 1974; (8) de Vaucouleurs 1978b; (9) Tully 1987; (10) Hutchmeier \& Richter 1986; (11) Bottinelli et al 1990; (12) Wevers et al 1986; (13) Masegosa et al 1991; (14) Skillman et al 1989; (15) González-Delgado et al 1994 .

this paper, we present the results of our photometric analysis of NGC 2366 (DDO 42, Markarian 71). Its coordinates are listed in Table 1, together with a summary of the properties of the galaxy. This galaxy is usually assumed to belong to the M81-NGC 2403 group (see Sandage \& Tammann 1974), whose distance, one fourth of that to the Virgo cluster, have 
TABLE 2. Journal of observations.

\begin{tabular}{ccccc}
\hline \hline Date & Time (UT) & Filter & Exp. time (s) & FWHM (") \\
\hline March 21, 1992 & $20: 33$ & $\mathrm{~V}$ & 2100 & 1.4 \\
March 21, 1992 & $21: 22$ & $\mathrm{R}$ & 1800 & 1.5 \\
March 21, 1992 & $22: 13$ & $\mathrm{~B}$ & 3500 & 2.0 \\
\hline
\end{tabular}

made the members of this group particularly interesting for the calibration of the extragalactic distance scale.

In closer relation with the present work, previous photometric data for the brightest stars of NGC 2366 have been published by Sandage \& Tammann (1974) and Tikhonov et al. (1991), both based on photographic photometry. These are the only photometric studies on the resolved stars in NGC 2366 carried out up to now, and no previous CCD photometry has been performed.

The present paper is divided in seven sections. Section 2 presents the observations and discusses the data reduction procedures, the photometric errors, and the completeness of the analyzed stellar sample. The comparison with previous photographic data for this galaxy is carried out in Sec. 3. A description of the features appearing in the photometric color-magnitude (CM) diagrams and the cleaning of these diagrams from foreground stars are discussed in Sec. 4 . The problem of the distance to NGC 2366 is discussed in Sec. 5. A discussion of the spatial distribution of stars is given in Sec. 6. Finally, the conclusions are summarized in Sec. 7.

\section{OBSERVATIONS, DATA REDUCTION, ERRORS, AND COMPLETENESS}

Observations of the stellar content of NGC 2366 were carried out in 1992 March using the $1150 \times 1250$ EEV5 chip at the prime focus of the $2.5 \mathrm{~m}$ Isaac Newton Telescope of the Observatory of Roque de los Muchachos in La Palma (Canary Islands, Spain). The EEV5 is a thick coated chip, with pixel size $22.5 \mu \mathrm{m}$, equivalent to 0.55 . The total field is about $10.5 \times 11.5 \operatorname{arcmin}^{2}$. Under very good seeing conditions these parameters might lead to undersampling and compromised photometric accuracy. In our case, seeing conditions were poor (see Table 2), and problems of undersampling are not apparent. Gallart et al. (1995) presented a detailed discussion on this issue, and the reader is referred to their paper for more information.

Table 2 shows the journal of observations, including, in the last column, the full width at half of maximum (FWHM) of the stellar profiles, measured in each frame. FIGARO, DAOPHOT, and ALLSTAR (Stetson 1987) were used on a VAX cluster of the Instituto de Astrofísica de Canarias to produce the photometry of the stars.

The usual procedure to transform the instrumental magnitudes derived from the Point Spread Function (PSF) fitting into aperture ones was used. We followed the same procedure described by Aparicio et al. (1993) (see Stetson 1987 for a full explanation of the foundations of the method), using 12 isolated stars for $B, 23$ for $V$, and 17 for $R$. We obtained the following errors for the transformations: 0.015 in $B, 0.018$ in $V$, and 0.07 in $R$.

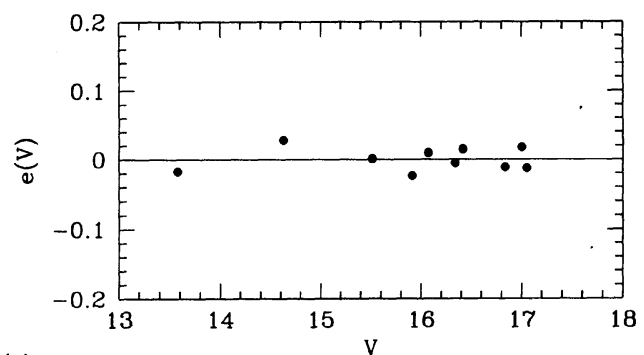

(a)
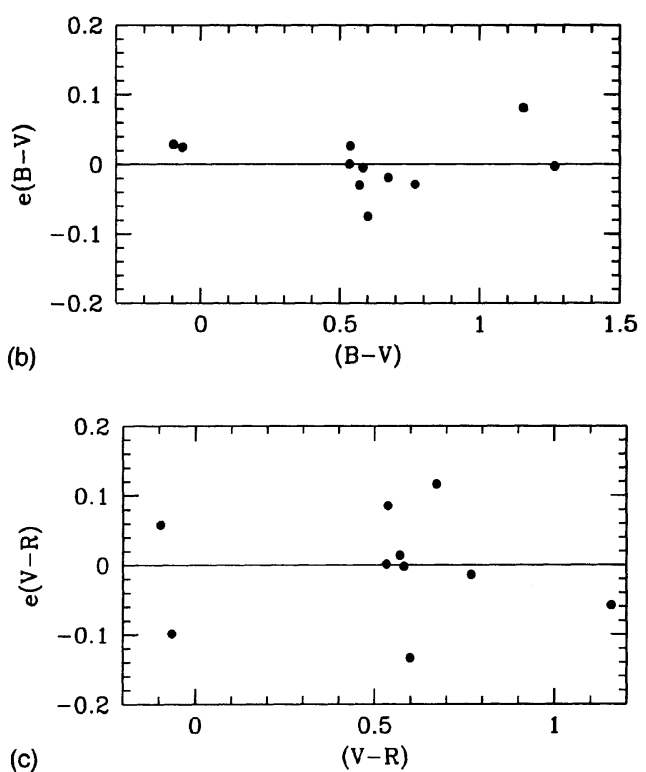

FIG. 1. (a) Photometric residuals vs magnitude of the calibration in the $V$ band. (b) Photometric residuals vs color index of the calibration in $(B-V)$. (c) Photometric residuals vs color index of the calibration in $(V-R)$.

Atmospheric extinction corrections for each night, and instrumental to Johnson-Cousins system transforming equations for the whole campaign were then determined. To this purpose, 45 measurements of a total of 11 secondary standards of the fields of the clusters M92 and NGC 2419 (see Christian et al. 1985) were taken in each band during the three nights of the campaign. The color indices covered by the standard stars were $-0.096<(B-V)<1.269$ and -0.040 $<(V-R)<0.803$. After correcting for atmospheric extinction, we derived the following set of equations transforming into the Johnson-Cousins standard system.

$$
\begin{aligned}
& V=v+24.033+0.048(B-V), \\
& (B-V)=-0.634+0.929(b-v), \\
& (V-R)=-0.363+0.992(v-r),
\end{aligned}
$$

where capital letters are Johnson-Cousins magnitudes and small letters are instrumental magnitudes. Figure 1 shows the residuals for each transformation as a function of the magnitude [Fig. 1(a)] and the color indices [Figs. 1(b) and 1(c)]. The corresponding zero-point errors are 0.014 in $V$ and $(B$ $-V$ ) and 0.025 in $(V-R)$. Adding quadratically this errors to those of the PSF to aperture magnitudes transformation, 
TABLE 3. Photometry.

\begin{tabular}{|c|c|c|c|c|c|}
\hline$N$ & $X$ & $Y$ & $(B-V)$ & $V$ & $(V-R)$ \\
\hline 1 & 8.3 & 759.7 & - & 20.66 & 1.14 \\
\hline 2 & 13.5 & 285.7 & - & 22.59 & 0.70 \\
\hline 3 & 18.0 & 5.6 & 1.18 & 17.66 & 0.61 \\
\hline 4 & 19.0 & 521.9 & - & 22.05 & 0.65 \\
\hline 5 & 25.6 & 380.0 & 0.72 & 17.83 & 0.29 \\
\hline 6 & 26.4 & 791.6 & 1.47 & 19.39 & - \\
\hline 7 & 28.9 & 310.2 & 0.65 & 17.11 & 0.24 \\
\hline 8 & 31.3 & 32.5 & - & 22.53 & 1.14 \\
\hline 9 & 32.9 & 63.3 & 0.92 & 17.98 & 0.40 \\
\hline 10 & 33.8 & 115.8 & - & 22.47 & 0.98 \\
\hline 11 & 35.0 & 93.2 & - & 22.55 & 0.01 \\
\hline 12 & 36.2 & 368.1 & - & 22.71 & 0.50 \\
\hline 13 & 36.6 & 455.7 & - & 21.68 & 0.85 \\
\hline 14 & 37.3 & 12.0 & - & 21.85 & 0.55 \\
\hline 15 & 38.0 & 314.4 & 1.61 & 20.82 & 0.76 \\
\hline 16 & 38.1 & 679.4 & - & 22.74 & 0.64 \\
\hline 17 & 38.2 & 733.7 & 0.97 & 21.57 & - \\
\hline 18 & 38.7 & 855.5 & 1.40 & 19.94 & - \\
\hline 19 & 39.0 & 771.0 & 1.18 & 17.61 & - \\
\hline 20 & 40.6 & 28.2 & - & 22.88 & 0.14 \\
\hline 21 & 40.7 & 246.9 & - & 22.73 & 1.26 \\
\hline 22 & 41.0 & 907.3 & - & 22.40 & 0.22 \\
\hline 23 & 42.6 & 644.0 & 0.91 & 17.54 & 0.34 \\
\hline 24 & 42.6 & 64.0 & - & 22.45 & -0.02 \\
\hline 25 & 48.3 & 67.5 & - & 21.20 & 0.78 \\
\hline 26 & 50.6 & 17.9 & - & 22.81 & 0.66 \\
\hline 27 & 55.2 & 311.4 & - & 21.33 & 0.42 \\
\hline 28 & 56.1 & 295.4 & 0.64 & 16.95 & 0.27 \\
\hline 29 & 61.9 & 688.5 & 0.60 & 18.89 & - \\
\hline 30 & 62.7 & 32.7 & - & 21.21 & 0.36 \\
\hline 31 & 64.2 & 281.3 & 1.34 & 21.38 & 1.05 \\
\hline 32 & 65.2 & 34.6 & - & 21.80 & 0.52 \\
\hline 33 & 67.8 & 14.2 & - & 22.02 & 0.73 \\
\hline 34 & 69.1 & 313.6 & - & 22.31 & 0.32 \\
\hline 35 & 69.4 & 513.1 & 1.31 & 18.14 & 0.59 \\
\hline 36 & 69.7 & 29.9 & 0.82 & 20.63 & 0.33 \\
\hline 37 & 71.2 & 862.8 & 1.73 & 20.14 & 0.93 \\
\hline 38 & 75.2 & 52.0 & - & 22.77 & 0.41 \\
\hline 39 & 76.2 & 636.6 & - & 21.94 & 0.84 \\
\hline 40 & 80.3 & 361.1 & 0.47 & 15.40 & 0.40 \\
\hline 41 & 82.2 & 678.7 & - & 21.17 & 0.41 \\
\hline
\end{tabular}

the final total zero point errors result: 0.02 in $V, 0.03$ in $(B-V)$, and 0.03 in $(V-R)$.

Our final photometric list is given in Table 3 , available in the ApJ-AJ CDROM series. This table contains 1959 stars with photometry in $V$ and, at least, one more band. Only stars with ALLSTAR's sigma values smaller than 0.2 have been considered. Column 1 lists an identification number for each star; columns 3 and 4 give the coordinates in pixels, columns 5 to 7 , respectively, list the $(B-V)$ color index, the $V$ magnitude and the $(V-R)$ color index. Figure 2 shows the finding chart of the stars given in Table 3. Coordinates are given in pixels and correspond to those listed in this table. The sizes of the circles are proportional to the $V$ magnitudes. The frame has been divided into several regions for its analysis. Five regions correspond to particular fields in the main body of NGC 2366 . The thick line shows the boundary between the full body adopted for the galaxy and the foreground field, of galactic stars. The division of the galaxy in five sub-fields will be used later, in Sec. 6, to discuss the spatial distribution of stars in NGC 2366.
The errors affecting the stellar photometry in crowded fields as well as the completeness factors, have been discussed in a number of papers (see, for example, Stetson 1987 and Chiosi et al. 1989). In the present work, crowding have been analyzed following the usual technique, based on artificial stars trials. The field of the CCD used is large enough to contain the whole galaxy and a companion field together. Since the crowding effects are different for the galaxy and the field, the artificial stars trials have to be carried out separately or, at least, in such a way as to reach results statistically significant for both (in particular for the galaxy, that covers a smaller area) and, at the same time, to avoid overcrowding effects. For this reason, a large number of trials with few artificial stars each have been carried out. In summary, about 1000 artificial stars have been added to each frame, distributed in 38 proofs per frame. We have derived the total errors of the photometry and the completeness factors in the same way described in Aparicio et al. (1993), for the galaxy and the companion field. The errors, estimated in this way, are less than 0.01 mag for the three filters at magnitude 19.0 At magnitude 22 the errors for the stars in the area of the galaxy are 0.10 in $B, 0.11$ in $V$, and 0.15 in $R$. For the stars of the surrounding field, they are 0.08 in $B, 0.04$ in $V$, and 0.08 in $R$. In Fig. 3, the resulting completeness factors as a function of the $B, V$, and $R$ magnitudes are plotted for the areas 3, 4, and 5 together (see Fig. 2) and for the companion field (only for $V$ ). According to them, at $50 \%$ level of completeness, and for stars in the area of the galaxy, we reach magnitudes $B=22.0, V=23.0$, and $R=22.8$. For comparison, the magnitude reached in the external companion field at the same level of completeness is $V=23.5$.

\section{COMPARISON WITH PREVIOUS PHOTOMETRIC DATA}

Sandage \& Tammann (1974) and Tikhonov et al. (1991) have carried out photographic photometry of resolved stars in NGC 2366. Calibration of photographic photometry usually shows systematic effects. However, it is interesting to test whether our photometry is consistent with the scales that these authors derived from their photoelectric sequences. In Table 4 magnitudes are listed for the stars of the sequence of Sandage \& Tammann (1974) that are not saturated in, at least, one of our frames. Column 1 lists the identification letter from Sandage \& Tammann (1974). Columns 2 and 3 show their photometric results. Columns 4 and 5 list the photometry by Tikhonov et al. (1991). These authors give photoelectric magnitudes for two stars of the sequence of Sandage \& Tammann (1974) (stars C and E). For the remainder stars, their photografic magnitudes are given. Finally, in columns 6 and 7, our results are listed for the stars not saturated.

Figure 4(a) shows the Sandage \& Tammann's (1974) $B$ and $V$ magnitudes, plotted against ours. The differences between our photometric scale and that of Sandage \& Tammann (1974) lie within the dispersion of the data. However, some systematic color effect might be present. Figure 4(b) shows the Tikhonov et al.'s (1991) $B$ and $V$ magnitudes of the Sandage and Tammann's photoelectric sequence, plotted against ours. In both cases, the agreement is good, in particu- 


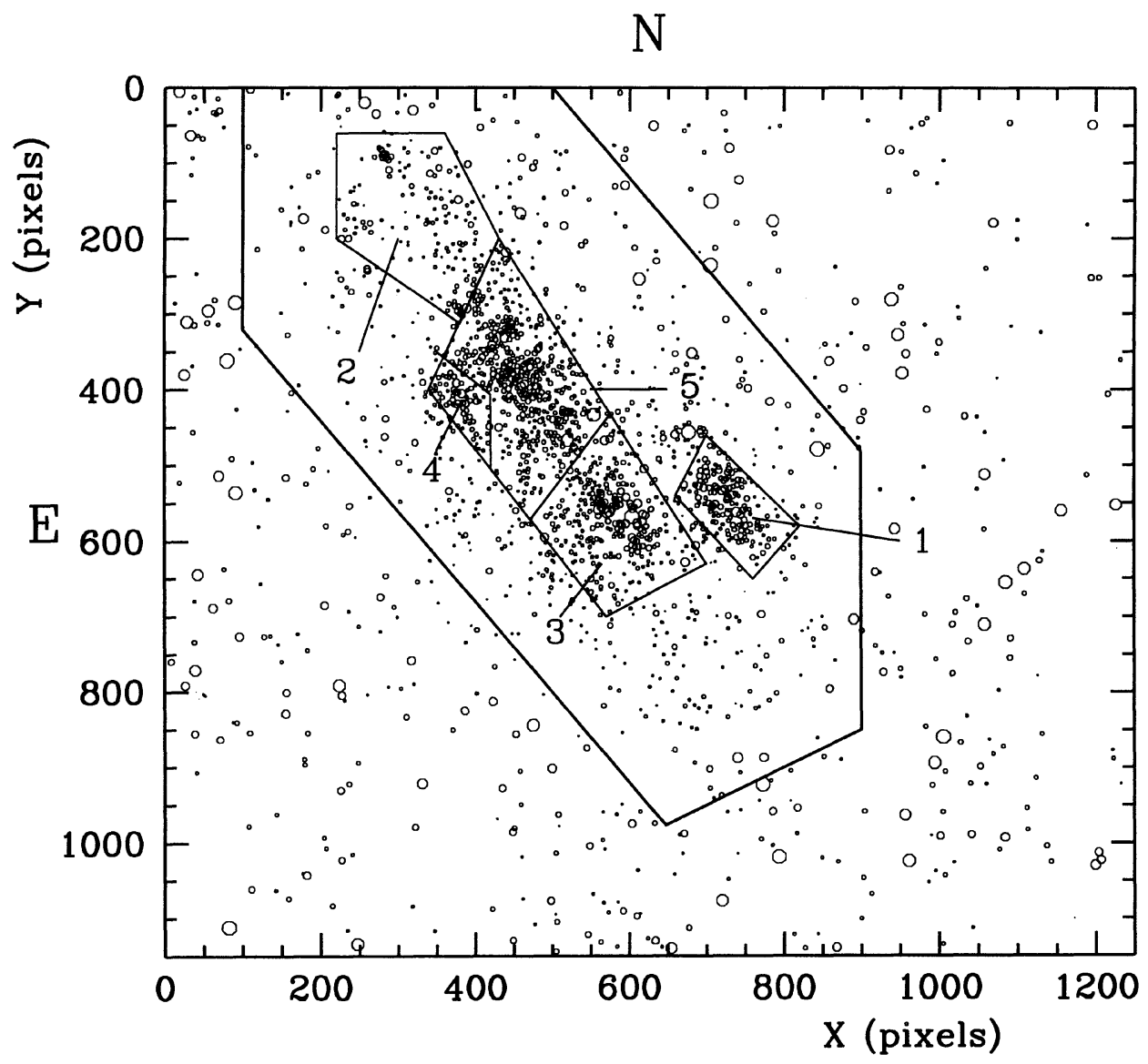

FIG. 2. Finding chart of the stars measured in NGC 2366 and the surrounding field. Coordinates are CCD pixels. The circles' sizes are proportional to the stars' $V$ magnitudes. The five areas in which the galaxy has been divided for the discussion of the stellar content (see Sec. 6) are shown. The boundary of the surrounding companion field, used to correct the contamination by foreground stars, is defined as the area outside the heavy line. North is up, East is at left.

lar for the magnitudes photoelectricity obtained by Tikhonov et al. (1991). But this is not the case when magnitudes of the Tikhonov et al.'s main list (their Table 3) are compared with ours. The results are shown in Fig. 4(c) for $B$ and $V$. Large

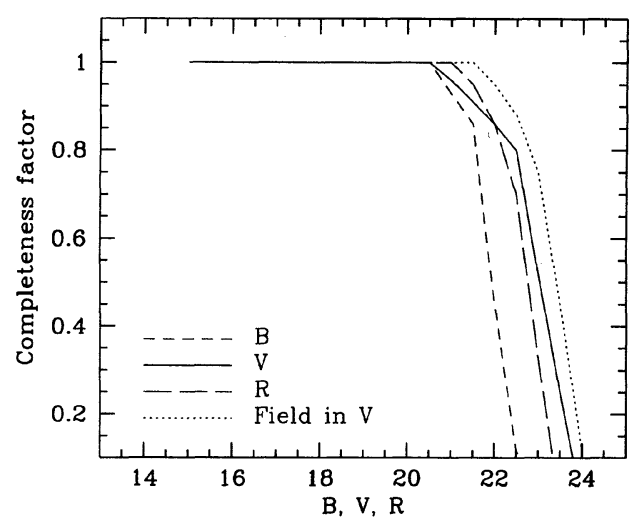

Fig. 3. Completeness factor as a function of $B, V$, and $R$ magnitudes in the central part of the galaxy (zones 3,4 , and 5 of Fig. 2) and the companion field (only in $V$ ). At 50\% completeness level, $B=22.0, V=23.0$, and $R=22.8$ are reached in the galaxy and $V=23.5$ is reached in the companion field. zero point differences exist in both cases and the dispersion of the data is also apparent. The fact that photographic photometry is usually noisier than photoelectric or CCD photometry is probably the reason for this disagreement.

\section{PHOTOMETRIC DIAGRAMS}

Figures 5(a) and 5(b) show the $V$ vs $(B-V)$ and $V$ vs $(V-R)$ color-magnitude (CM) diagrams for the stars in the full area of the galaxy (the zone internal to the thick line in Fig. 2). Contamination by foreground Milky Way stars is expected to be important due to the low galactic latitude of NGC 2366 (see Table I). This fact is visible in Figs. 6(a) and

TABLE 4. Comparison with previous photoelectric sequences.

\begin{tabular}{ccccccc}
\hline \hline Star & $B_{S T}$ & $V_{S T}$ & $B_{T B K G}$ & $V_{T B K G}$ & $B_{\text {here }}$ & $V_{\text {here }}$ \\
\hline B & 13.94 & 13.03 & - & - & 14.28 & - \\
C & 14.43 & 13.76 & 14.49 & 13.70 & 14.53 & - \\
D & 15.79 & 15.21 & - & - & 15.91 & 15.37 \\
E & 18.04 & 16.87 & 18.02 & 16.74 & 18.00 & 16.68 \\
F & 19.63 & 18.63 & $19.82(\mathrm{pg})$ & $18.74(\mathrm{pg})$ & 19.70 & 18.50 \\
G & 20.16 & 19.07 & $20.10(\mathrm{pg})$ & $19.03(\mathrm{pg})$ & 20.10 & 18.86 \\
H & 19.75 & 19.28 & $19.89(\mathrm{pg})$ & $19.18(\mathrm{pg})$ & 19.84 & 18.91 \\
I & 17.64 & 16.98 & $17.58(\mathrm{pg})$ & $16.92(\mathrm{pg})$ & 17.78 & 17.04 \\
\hline
\end{tabular}



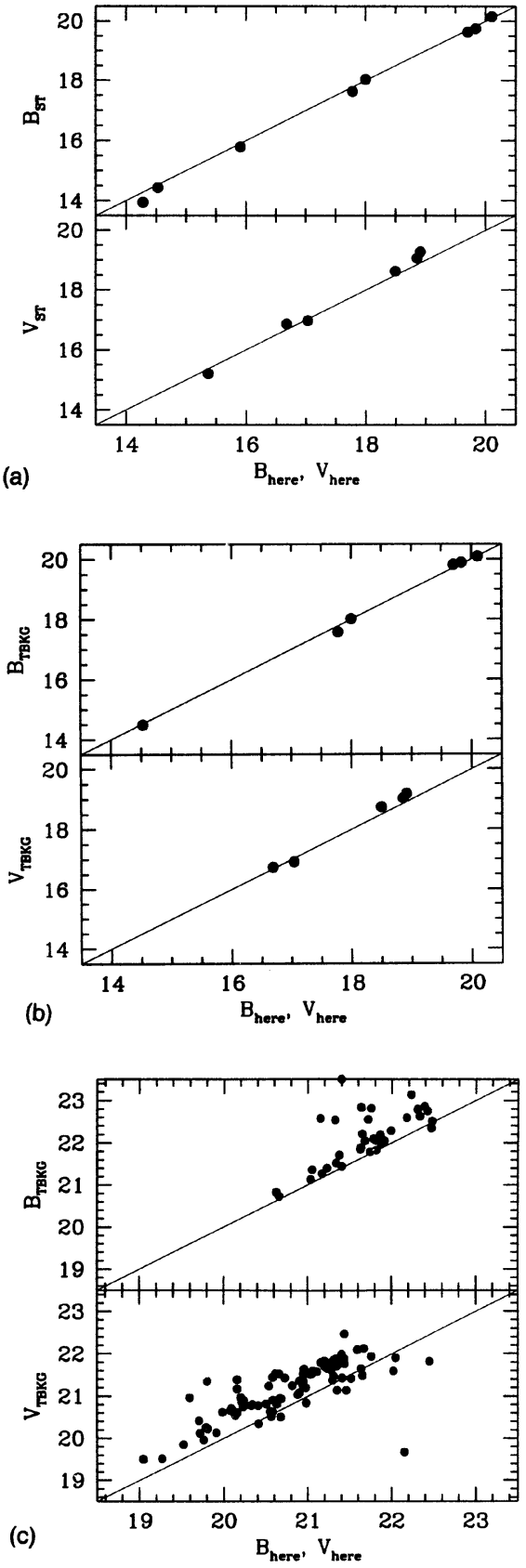

Fig. 4. (a) Photoelectric $B$ and $V$ magnitudes of the stars of the calibration sequence of Sandage \& Tammann (1974) plotted against our data. (b) Photoelectric and photographic $B$ and $V$ magnitudes of the stars of the calibration sequence of Tikhonov et al. (1991) plotted against our data. (c) Photographic $B$ and $V$ magnitudes of the stars measured by Tikhonov et al. (1991) in NGC 2366, plotted against our data. 87 stars have been matched.

6(b), where the CM diagrams of stars of the companion field (the zone external to the thick line in Fig. 2) are plotted.

To decontaminate the CM diagrams of NGC 2366 from foreground stars, a procedure similar to that explained by Mateo \& Hodge (1986) has been followed. This procedure is based on randomly removing stars from the $\mathrm{CM}$ diagram of the galaxy, but in such a way that the total number of stars removed in each area of the diagram be equal to the total number of stars in the companion field, after correcting to

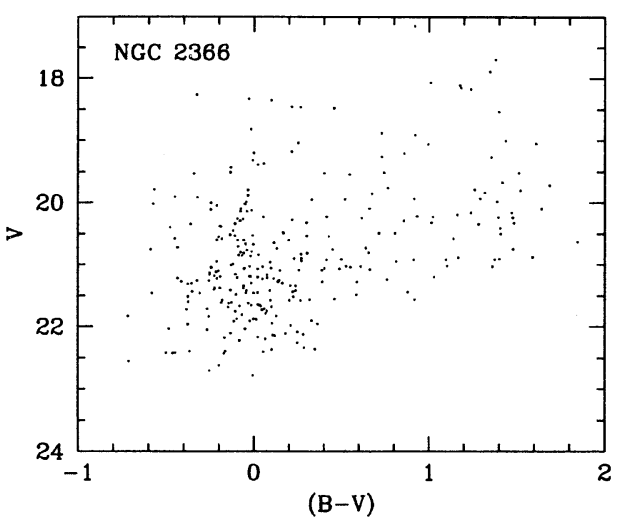

(a)

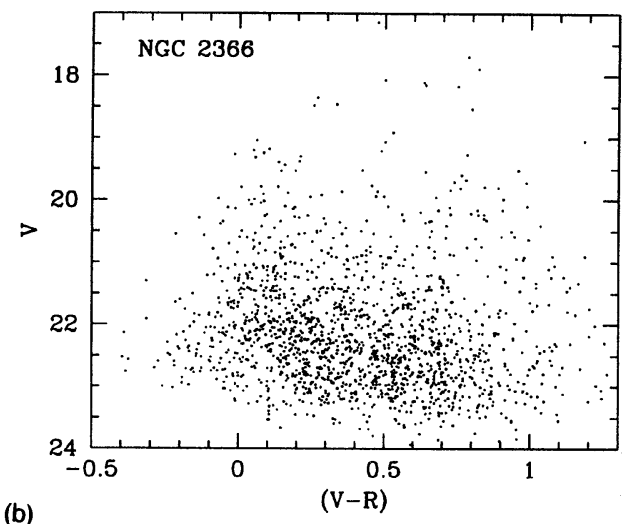

FIG. 5. The $V$ vs $(B-V)$ CM diagram of the stars in the area of NGC 2366 (see Fig. 2). (b) The $V$ vs $(V-R)$ CM diagram of the stars in the area of NGC 2366 (see Fig. 2).

compensate for the different surface of galaxy and field and for the crowding effects, which are different in the galaxy and in the field. The resulting CM diagrams are shown in Figs. 7(a) and 7(b). Note, in particular, that virtually all the stars brighter than $V \simeq 19.0$, have been removed.

The CM diagrams of NGC 2366 are typical of a galaxy with active star formation. They are similar to those of other Im galaxies, e.g., Sextans A (Aparicio et al. 1987), NGC 3109 (Bressolin et al. 1993; Greggio et al. 1993), WLM (Ferraro et al. 1989) or NGC 6822 (Gallart et al. 1994; Gallart et al. 1995). The diagrams of these galaxies are usually characterized by the presence of two main plumes separated by a scarcely populated region. The two plumes are not clearly visible in NGC 2366 . The blending produced by the poor seeing together with the distance to the galaxy, larger than that of the Local Group members, is very likely the reason for this confusion. In any case, the region corresponding to the blue plume is placed at $(B-V)$ about -0.3 to 0.3 , $(V-R)$ about -0.15 to 0.35 and the region corresponding to the red plume is placed at $(B-V)$ about 1.0 to $1.5,(V-R)$ about 0.6 to 0.9 .

At the distance of NGC 2366, the limiting magnitudes that we have reached imply that our photometry extend only to stars brighter than $M_{V} \simeq-4.5, M_{V_{0}} \simeq-5$ (50\% completeness level) in the $V$ vs $(V-R)$ diagram, which is that with 


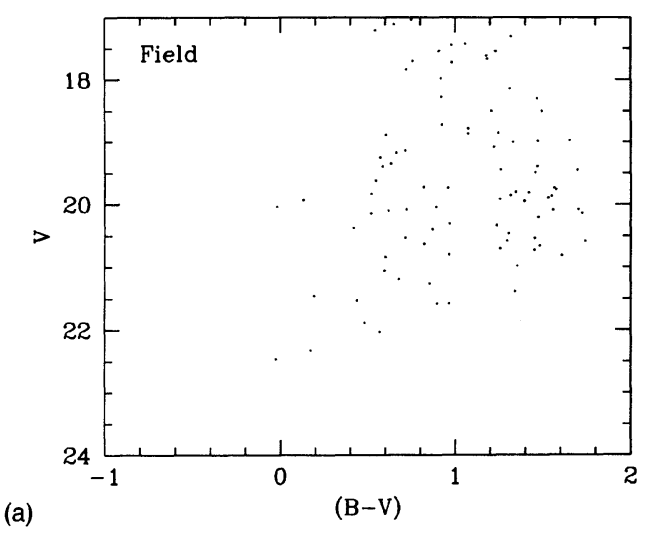

(a)

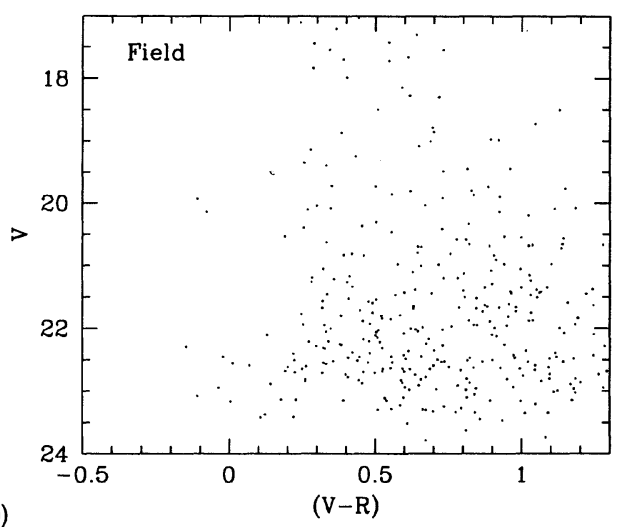

(b)

FIG. 6. (a) The $V$ vs $(B-V)$ CM diagram of the stars in the surrounding companion field of NGC 2366 (see Fig. 2). (b) The $V$ vs $(V-R)$ CM diagram of the stars in the surrounding companion field of NGC 2366 (see Fig. 2).

the best completeness. As a first approximation, using data for bolometric corrections from Schmidt-Kaler (1982), it results in a luminosity of $\log \left(L / L_{\odot}\right) \simeq 4.2$. This means that we could barely detect some red, intermediate-mass stars of masses down to about $5 M_{\odot}$; but, certainly, no low or intermediate mass star of the asymptotic or red giant branches can be present in our diagram contrary to what happens in NGC 6822 (Gallart et al. 1994,1995) and Pegasus (Aparicio 1994; Aparicio \& Gallart 1995). The problem is more complicated for stars in the blue plume. Our limiting magnitude implies that we are observing main-sequence stars down to only $\mathrm{O} 9$ (over $15-20 M_{\odot}$ ). However, most of the stars of the blue plume are probably already evolved away from the main sequence, and a number of stars in the red edge of the blue plume might be less massive stars in their blue-loop evolutionary phase. In any case, the bolometric corrections that affect this part of the CM diagram are large and they change quickly as a function of the spectral type. This complicates the determination of the spectral type of each star as well as the analysis of the blue stellar population. In Sec. 6, we shall discuss in more detail the age of the stellar populations in NGC 2366.
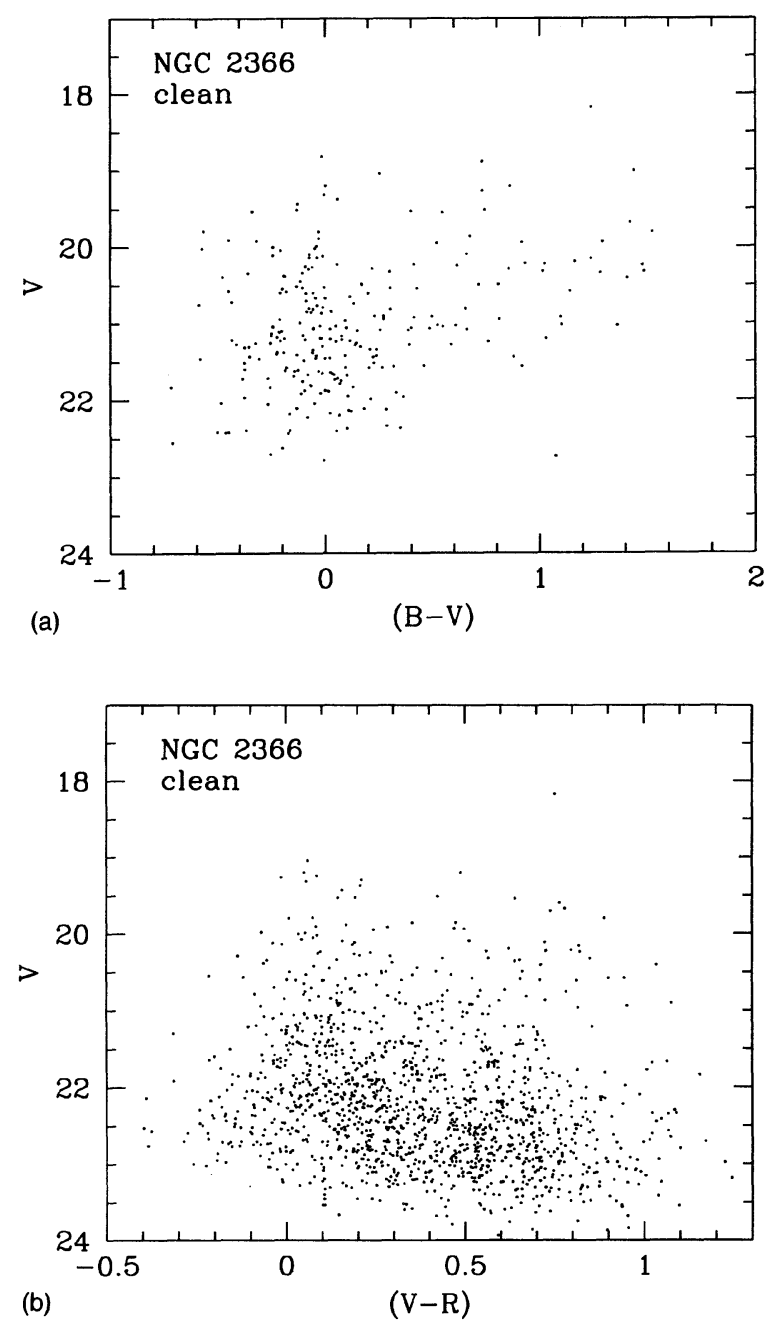

FIG. 7. (a) The $V$ vs $(B-V)$ CM diagram of the stars of NGC 2366 after correction of contamination by foreground stars. (b) The $V$ vs $(V-R)$ CM diagram of the stars of NGC 2366 after correction of contamination by foreground stars.

\section{THE DISTANCE TO NGC 2366}

The M81/NGC 2403 group, to which NGC 2366 probably belongs, is placed at a distance $(3-4 \mathrm{Mpc})$, that makes it of great importance for the calibration of secondary distance indicators. A large number of papers have been published about this subject (see de Vaucouleurs 1978b, and references therein). Freedman et al. (1994) have recently published the light curves of 30 cepheids stars in M81, obtained with the Space Telescope. They derive a distance to this galaxy of 3.63 Mpc. But few cepheid stars have been discovered and measured until now in the other galaxies of this group; in particular, none in NGC 2366.

Estimates of the true distance modulus of NGC 2366 that are found in the literature range from 27.07 to 27.65 . Three of them are based on the blue supergiants: Sandage \& Tammann (1974) found a value of 27.07; de Vaucouleurs (1978b) found 27.10, and Tikhonov et al. (1991) found 27.65. Using the size of the H II regions, Sandage \& Tammann (1974) found 27.07. Finally, Tikhonov et al. (1991) found 27.62, 
TABLE 5. Distance estimates to NGC 2366.

\begin{tabular}{ccl}
\hline \hline Estimator & $(m-M)_{0}$ & Reference \\
\hline Blue supergiants & 27.07 & Sandage \& Tammann (1974) \\
$"$ & 27.10 & de Vaucouleurs (1978b) \\
$"$ & 27.65 & Tikhonov et al (1991) \\
Red supergiants & 27.00 & This paper \\
" & 27.62 & Tikhonov et al (1991) \\
HII regions & 27.60 & This paper \\
Local velocity field & 27.07 & Sandage \& Tammann (1974) \\
& 27.35 & Tully (1987) \\
\hline
\end{tabular}

from the brightest red supergiants. A further value was provided by Tully (1987) based on the local velocity field: using $H=75 \mathrm{Km} \mathrm{s}^{-1} \mathrm{Mpc}^{-1}$, he derived $(m-M)_{0}=27.35$.

We can provide a new estimate of the distance modulus using our data and the method of the brightest blue and red supergiants in the galaxy. The problems of this method have been discussed by several authors, and the papers by Schild \& Maeder (1983) and by Greggio (1986) are very enlightening. But, till now, it is one of the few ways we have to estimate the distance to a large number of objects, NGC 2366 being among them. The average $B$ magnitude of the three brightest blue stars [using the criterion of $(B-V) \leqslant 0.5$ ] is $\langle B\rangle_{3}=19.17$. The average $V$ magnitude of the three brightest red ones [with $(B-V) \geqslant 1.5$ ] is $\langle V\rangle=20.15$. Using data given by de Vaucouleurs et al. (1991) for the interstellar extinction and the integrated magnitude of the galaxy and Eq. (5) from de Vaucouleurs (1978a), we derive a true distance modulus for NGC 2366 of $(m-M)_{0}=26.99$ from the blue stars. On the other hand, using the result by Sandage \& Tammann (1974) that the average absolute magnitude for the three brightest red supergiants in a galaxy is $\left\langle M_{V}\right\rangle_{3}=-7.7$, we obtain for NGC 2366, from the red stars, $(m-M)_{0}=27.49$. Alternatively, using the fits given by Aparicio et al. (1987), we obtain $(m-M)_{0}=27.01$ from the blue supergiants and $(m-M)_{0}=27.58$ from the red supergiants. In the following, we will adopt the value $(m-M)_{0}=27.3$, which is an average of the four estimates. It corresponds to $2.9 \mathrm{Mpc}$. A summary of the different estimates found in the literature is given in Table 5 .

The question of the distance to this galaxy is still open, and the discovery of cepheid stars in it would be quite important. The comparison between the Tikhonov et al.'s magnitudes and ours would be very useful to make a first list of cepheid candidates. Unfortunately, the dispersion of the data [see Fig. 4(c)] does not allow for any step in this direction.

\section{THE SPATIAL DISTRIBUTION OF STARS IN NGC 2366}

The morphology of NGC 2366, patched by a number of $\mathrm{H}$ II regions, indicates that star formation is ongoing in many regions of the galaxy. In particular, NGC 2366 includes a giant $\mathrm{H}$ II region, named NGC 2363, placed in the SouthWest part of the galaxy. The GEFE collaboration (GonzálezDelgado et al. 1994) has recently carried out a detailed study of the physical conditions and chemical composition of this giant $\mathrm{H}$ II complex.

To test the possible differences in the star forming activity across NGC 2366, we have divided the galaxy into five re-
TABLE 6. Integrated magnitudes and colors.

\begin{tabular}{cccc}
\hline \hline Zone & $V_{0}^{\text {int }}$ & $(B-V)_{0}^{\text {int }}$ & $(V-R)_{0}^{\text {int }}$ \\
\hline 1 & 22.95 & 0.06 & 0.07 \\
3 & 22.14 & 0.07 & 0.09 \\
4 & 22.83 & 0.00 & -0.02 \\
5 & 22.39 & 0.12 & 0.15 \\
\hline
\end{tabular}

gions. These are shown in Fig. 2. Four regions (2, 3, 4, and 5) have been defined along the bar and a further one in South-West (region 1). Region 4 has been differentiated from region 5 because of its apparently higher current star forming activity. The integrated magnitude and color indices of these regions together with the population of their $\mathrm{CM}$ diagrams provide valuable information about the general distribution of the stellar population in the galaxy.

Table 6 lists the integrated $V_{0}$ magnitude and $(B-V)_{0}$ and $(V-R)_{0}$ color indices (columns 2 to 4 ) for each of the regions defined in Fig. 2 (column 1). Magnitude and color indices have been obtained from sky-subtracted calibrated frames, so that resolved and unresolved galaxy light is included in the results. These have been corrected for internal and galactic extinction using $A_{B}=0.47, A_{V}=0.35, A_{R}=0.26$. These values have been obtained using $A_{B}=0.47$ given by de Vaucouleurs et al. (1991) (see Table 1) and the Cardelli et al.'s (1989) extinction law, with $R_{V}=3.1$. Region 2 has been omitted because of the low signal-to-noise ratio.

A first look at Table 6 shows that all the regions we have defined have $(B-V)_{0}$ between 0.00 and 0.12 . A typical low metallicity burst of star formation at ages between 2 and 5 Myr [González-Delgado et al. (1994) have derived an age of about 3-5 Myr for NGC 2363 from WR features] would show $(B-V)_{0}$ close to 0.0 (Leitherer \& Heckman 1995). Note that the integrated colors of regions dominated by an intense star formation episode can depart significantly from the colors of the stellar population alone, due to the contribution of the nebular continuum emission. For NGC 2363, for example, this contribution can account to up to $50 \%(V)$ and $70 \%(R)$ of the continuum, as shown by Mas-Hesse \& Kunth (1991). This contribution is already included in the model predictions by Leitherer \& Heckman (1995). Therefore, only region 4 could be truly dominated by blue stars formed during the last burst of star formation. All the other regions show $(B-V)_{0}$ color indices redder than expected for a very young star formation episode. Comparing with the $(B-V)_{0}$ predictions of Leitherer \& Heckman (1995) we see that the integrated $(B-V)_{0}$ of these regions are consistent with star forming episodes having taken place some $20-50$ Myr ago. It is interesting to note that older stars would yield significantly redder color indices, so that it seems that a major event of star formation took place along the whole galaxy some few tens of million of years ago.

The results listed in Table 6 can be also compared with data given in Table 1 for the whole galaxy. From this table, $(B-V)_{0}^{b i} \simeq 0.46,(V-R)_{0}^{b i}=-0.12$ are obtained. $(V-R)_{0}^{b i}$ is too blue compared with $(B-V)_{0}^{b i}$. The reason for this is perhaps an inconsistency between magnitudes in the three filters, which have been obtained by different authors. If we limit to data by Pierce \& Tully (1992), then $(B-R)_{0}^{b i}=0.29$. 
from Bertelli et al. (1994). Isochrones ages are 6, 10, 20, 40, and $100 \mathrm{Myr}$, from top to bottom. The diagrams show that stars are concentrated around the isochrones corresponding to about 40 Myr. Many of these stars, with $M_{V_{0}}$ around -5 to -6 , are presently in the He burning phase. The diagrams do not provide clear information for older, fainter stars, which are not resolved. However, the age value of $40 \mathrm{Myr}$ agrees with the results derived from the integrated $(B-V)$ colors.

Apart from this population of stars around $40 \mathrm{Myr}$ old the $\mathrm{CM}$ diagrams show the presence of much younger stars. $\mathrm{Re}$ gions 1 and 3 show the highest concentration of stars younger than $20 \mathrm{Myr}$; Region 3 contains several resolved stars younger than $6 \mathrm{Myr}$. Following the Bertelli et al.'s models stars of this age can have up to $34 M_{\odot}$, but Region 3 includes the giant $\mathrm{H}$ II region NGC 2363, and other younger, more massive stars have to be within this region, embedded into $\mathrm{H}$ II regions gass emission, and they have not been resolved. In fact, González-Delgado et al. (1994) have found WR features in NGC 2363 associated with the presence of WC stars. This implies ages between 3 and 5 Myr for the region. This has to be the case also of region 4 , whose blue integrated colors indicate the presence of a young star forming episode. Region 2, in the North-East part of the galaxy, is the less populated by very young stars.

The integrated color indices and the structure of the CM diagrams indicate that an age gradient may be present in the recent star formation episodes in NGC 2366, in the sense that the youngest population are preferentially placed in the South-West part of the bar. This picture is common to many Im galaxies and could indicate that a self-propagating mechanism is acting, triggering large scale star formation. If this is right, the velocity of the star formation propagation can be estimated. One could assume an age of $50 \mathrm{Myr}$ for the latest star formation event in region 2 and $2 \mathrm{Myr}$ for that of region 3 . These regions are separated by about 5 arcmin, and taking into account the distance to the galaxy (see Sec. 5) and the inclination angle (see Table 1) we obtain about 100 $\mathrm{Kms}^{-1}$ for the speed of propagation of stellar formation. This value seems too large when compared to the speed of propagating stellar formation $\left(\leqslant 30-50 \mathrm{Kms}^{-1}\right)$ in other galaxies (see Elmegreen 1992 and references therein). Consequently, self-propagation is probably not the main mechanism triggering the star formation at large scale in NGC 2366. In fact, self-propagation is not evident in the light of the CM diagrams of the five defined regions, since young stars are present in all of them: star formation is presently taking place preferentially in regions 1,3 , and 4 , but there are young stars also in regions 2 and 5 . Our conclusion is that large star formation bursts are produced in the galaxy without a physical connection between them. The bar however, seems to favor the star formation in one of its extremes.

\section{CONCLUSIONS}

The stellar content of the Im galaxy NGC 2366 has been discussed, through the study of the $V-(B-V)$ and $V-(V$ $-R)$ CM diagrams. A true distance modulus of $(m-M)_{0}=27.3$ has been obtained using the method of the brightest stars, in good agreement with most other estimates. But the determination of the distance through the light curve of cepheid stars is necessary in this galaxy, which belongs to a group of paramount importance in the calibration of secondary distance estimators.

A generalized star formation burst seems to have taken place about $50 \mathrm{Myr}$ ago. It would have been strong enough to dominate the integrated color indices of most of the galaxy. The spatial distribution of the young population indicates that young stars are preferentially concentrated in the South-West part of the bar structure, in particular in the NGC 2363 giant H II complex. Older stars populate the North-East part of the bar. A small region, populated by very young stars is placed at the East of the galaxy. The possibility that self-propagation could be the trigger of star formation at large scale is unlikely. We conclude that large bursts are produced at random positions, but the galactic bar, like in other Im galaxies, seems to play a role favoring star formation in one of its extremes.

This research has been funded by the spanish DGICyT (project reference: PB91-0531). We acknowledge the software provided by the Starlink Project which is funded by the UK SERC.

\section{REFERENCES}

Aparicio, A. 1994, ApJ, 437, L27

Aparicio, A., \& Gallart, C. 1995, AJ (submitted)

Aparicio, A., Alfaro, E., Delgado, A. J., Rodríguez-Ulloa, J. A., \& CabreraCaño, J. 1993, AJ, 106, 1547

Aparicio, A., García-Pelayo, J. M., Moles, M., \& Melnick, J. 1987, A\&AS, 71,297

Bertelli, G., Bressan, A., Fagotto, F., Chiosi, C., \& Nasi, E. 1994, A\&AS, 106,275

Bottinelli, L., Gouguenheim, L., Fouqué, P., \& Paturel, G. 1990, A\&AS, 82, 391

Bresolin, F., Capaccioli, M, \& Piotto, G. 1993, AJ, 105, 1779

Burstein, D., \& Heiles, C. 1984, ApJS, 54, 33

Cardelli, J. A., Clayton, G. C., \& Mathis J. S. 1989, ApJ, 345, 245

Chiosi, C., Bertelli, G., Meyland, G., Ortolani, S. 1989, A\&A, 219, 167

Christian, C. A., Adams, M., Barnes, J. V., Butcher, H., Hayes, D. S., Mould J. R., \& Siegel, M. 1985, PASP, 97, 363 de Vaucouleurs, G. 1978a, ApJ, 224, 14

de Vaucouleurs, G. 1978b, ApJ, 224, 710

de Vaucouleurs, G., de Vaucouleurs, A., Corwin, H. G., Buta, R. J., Paturel, G., \& Fouqué, P. 1991, Third Reference Catalogue of Bright Galaxies (Springer, Berlin)

Elmegreen, B. G. 1992, in Star Formation in Stellar Systems, edited by G. Tenorio-Tagle, M. Prieto and F. Sánchez (Cambridge University Press, Cambridge)

Ferraro, F. R., Fusi-Pecci, F., Tosi, M., \& Buonanno, R. 1989, MNRAS, 241, 433

Freedman, W. L., Hughes, S. M., Madore, B. F., \& Mould, J. R. 1994, ApJ, 427, 628

Gallart, C., Aparicio, A., Chiosi, C., Bertelli, G., \& Vílchez, J. M. 1994, ApJ, 425, L9

Gallart, C., Aparicio, A., \& Vílchez, J. M. 1995, in preparation

González-Delgado, R. M., et al. 1994, ApJ, 437, 239 
Greggio, L. 1986, A\&A, 160, 111

Greggio, L., Marconi, G., Tosi, M., \& Focardi, P. 1993, AJ, 105, 894

Hutchmeier, W. K., \& Richter, O. G. 1986, A\&AS, 63, 323

Kraan-Korteweg, R. C., Cameron, L. M., \& Tammann, G. A. 1988, ApJ 331, 620

Leitherer, C., \& Heckman, T. M. 1995, ApJS, 96, 9 Mas-Hesse, M., \& Kunth, D. 1991, A\&AS, 88, 399

Masegosa, J., Moles, M., \& del Olmo, A. 1991, A\&A, 249, 505

Mateo, M., \& Hodge, P., 1986, ApJS, 60, 893

Pierce, M. J., \& Tully, R. B. 1992, ApJ, 387, 47

Sandage, A., \& Tammann, G. A. 1974, ApJ, 191, 603

Schild, H., \& Maeder, A. 1983, A\&A, 127, 238
Schmidt-Kaler, Th. 1982, Landolt-Börnstein, (Springer, Berlin), Group VI, Vol. 2b, p. 1

Shimasaku, K., \& Okamura, S. 1992, ApJ, 398, 441

Skillman, E. D., Kennicut, R. C., \& Hodge, P. W. 1989, ApJ, 347, 875

Stetson, P. B. 1987, PASP, 99, 191

Tikhonov, N. A., Bilkina, B. I., Karachentsev, I. D., \& Georgiev, Ts. B. 1991, A\&AS, 89, 1

Tully, R. B. 1987, Nearby Galaxies Catalog (Cambridge University Press, Cambridge)

Wevers, B. M. H. R., Van der Kruit, P. C., \& Allen R. J. 1986, A\&AS, 66, 502

Yahil, A., Tammann, G. A., \& Sandage, A. 1977, ApJ, 217, 903 